\title{
19.
}

\section{Mémoire sur l'équilibre intérieur des corps solides homogènes.}

(Par Mir. Lamé et Clapeyron, Colonels du Génie au service de Russie.)

\section{R a p p o r t}

fait par MMI. Poinsot et Navier à l'académie des sciences de Paris dans la séance du 29. Septembre 1828, sur un mémoire de MM. Lamé et Clapeyron, concernant l'équilibre intérieur des corps solides homogènes.

L'académie nous a chargés, M. Poinsot et moi, de lui rendre compte d'un mémoire présenté par MM. Lamé et Clapeyron, anciens élèves de l'École Polytechnique, ingénieurs des mines, actuellement au service de Russie. Ce mémoire, qui n'est pas le premier ouvrage qui lui ait été soumis par ces jeunes savans, a pour objet la recherche des conditions de léquilibre des corps solides élastiques, lorsque ces corps sont exposés à laction de diverses forces qui tendent à en changer la figure. On sait que les questions de ce genre ont attiré l'attention de plusieurs illustres géomètres, parmi lesquels on peut citer Leibnitz, Jacques Bernoulli, Euler et Lagrange. Néanmoins elles n'ont été traitées jusqu'à présent (qu'en admettant certaines hypothèses qui conviennent seulement ì des cas particuliers et fort restreints, et d'après lesquelles on n'a pu se former des notions suffisamment exactes et étendues sur la nature des phénomènes qui sont dus à cette propriété physique des corps solides désignée sous le nom d'élasticité. MM. Lamé et Clapeyron ont voulu traiter les mêmes questions d'une manière générale, et exprimer au moyen du calcul les loix de ces phénomènes, qui sont du domaine de la physique et de lart des constructions, et qu'il importe beaucoup aux ingénieurs de connaitre et d'apprécier avec précision.

Les recherches de ce genre, dont on troure des modèles dans les théories du mouvement des fluides et de la distribution de la chaleur, comprennent ordinairement deux parties principales, On exprime dabord par les formules analytiques des conditions générales qui conviennent à um corps d'une figure arbitraire, sollicité par des forces également indétermi- 
nées; conditions qui dépendent seulement des loix connues de l'équilibre des forces, et de la nature physique du système que l'on considère. II faut ensuite particulariser ces résultats généraux, en les appliquant aux divers cas qui peuvent se présenter, et obtenir, pour chacun de ces cas, la connaissance des effets qui sont l'objet de la recherche, en ayant égard en même temps aux conditions générales, ì la figure particulière du corps, et aux forces données auxquelles il est soumis. Si l'on veut employer les expressions usitées par les géomètres, les deux opérations que nous venons d'indiquer consistent dans la recherche des équations différentielles propres à la question, et dans l'intégration de ces équations.

Quant à l'établissement des équations différentielles destinées à représenter les conditions générales de l'équilibre des parties des solides élastiques MM. Lamé et Clapeyron ont admis le même principe, et procédé de la même manière que l'un de nous l'avait fait dans un mémoire qui a été présenté à l'Académie en 1821, publié par extrait dans le Bulletin des sciences de la société philomatique en 1822, et imprimé en entier l'année dernière dans le tome VII. de nos mémoires. Ils parviennent à des équations semblables à celles qui avaient été données dans cet écrit. On doit seulement remarquer qu'ils ont déduit directement du principe dont il s'agit les équations déterminées relatives aux points de la sı::face du corps, et qui font connaître aussi les pressions ou tensions intérieures; tandis que, dans le mémoire de 1821, ces équations avaient été obtenues par les méthodes de la mécanique analytique. Nous sommes obligés de rappeler ici que ce dernier mémoire contient la première idée de la question, et les fondements des recherches relatives aux corps élastiques. MM. Lamé et Clapeyron n'en faisant aucune mention, on doit penser qu'ils n'en avaient pas connaissance, et qu'ils sont parvenus de leur côté aux mêmes résultats. Ils ont d'ailleurs poursuivi avec succès le genre de recherches qui étoit l'objet de cet écrit.

Les auteurs nomment dilatation le rapport de la variation que subit le volume d'un élément du corps par l'effet du changement de figure, au volume primitif ou naturel de cet élément. Ils donnent l'expression de ce rapport en fonction des déplacemens des molécules, et une équation différentielle générale à laquelle cette expression doit satisfaire. On remarque que cette équation, dans le cas particulier où les forces appliquées aux points intérieurs du corps sont constantes ou nulles, ne diffère pas do 
l'éçuation ì laquelle doit satisfaire l'expression de la température des points d'un corps solide, lorsque le mouvement de la chaleur est devenu constant, et que cette température ne varie plus avec le tems.

MM. Lamé et Clapeyron ont étudié les loix générales des déplacements des molécules intérieures des corps élastiques, et des pressions ou tensions qui s'établissent entre leurs parties. Ils ont reconnu, d'une part qu'un élément du volume, dont la figure serait, dans l'état naturel du corps, une sphère d'un rayon très petit, se changeait toujours en un ellipsoïle; d'autre part, quil existoit toujours parmi les pressions intérieures trois pressions principales perpendiculaires entre elles, données par les racines d'une équation du troisième degré, et qui correspondent aux demidiamètres d'un autre ellipsoïde construit sur les mêmes axes que le promier. Les rayons vecteurs de ce dernier ellipsoïde représentent les pressions qui ont lieu autour du centre dans toutes les directions. Il existe de plus une troisième surface du second degré, construite sur les mêmes axes que ces deux ellipsoïdes, et telle que si l'on mène à cette surface un plan tangent, le rayon vecteur dirigé sur le point de tangence, et considéré comme appartenant au second ellipsoïle, représente la pression ou tension qui est exercée au centre commun sur un élément superficiel parallèle à ce plan tangent. Le mémoire contient les équations de ces surfaces, et la discussion des cas où les parties du corps étant pressées dans tous les sens, ou tirées dans un sens et pressées dans un autre, la dernière surface du second degré est un ellipsoïle, ou la réunion de deux hyperbolöiles conjuguées à une et à deux nappes. On voit ici des effets assujettis ì la loi de continuité liés entre eux, et représentés au moyen des propriétés des surfaces du second degré. Ces résultats, dont diverses théories appartenant à la géométrie et à la mécanique ont déjà donné des exemples, ont l'avantage de faire connaître completement, et sous des formes que l'esprit saisit avec facilité, les modifications subies par les parties intérieures des corps.

La partie des Exercices de mathématiques de Mr. Cauchy qui a été publiée l'année dernière contient plusieurs propositions relatives aux pressions intérieures qui ont lieu dans un corps solide, analogues aux précédentes, et dont quelques - unes avaient été données antérieurement par Mr. Fresnel. MM. Lamé et Clapeyron remarquent que la théorie exposée dans leur ourrage diffère essentiellement de celie qu'avait adoptée 
148 19. Lamé et Clapeyron, mém. sur l'équilibre int. des corps sol. homog.

Mr. Cauchy. Nous nous abstenons de parler des recherches qui ont été publiées après la présentation du mémoire qui est l'objet de ce rapport.

Après avoir donné les équations et les propositions générales qui appartiennent à la théorie des corps élastiques, les auteurs traitent diverses questions spéciales. Ils considèrent successivement un prisme soumis à une pression extérieure constante, et tiré dans le sens de sa longueur; un corps de forme quelconque pressé extérieurement; un cylindre soumis également $\grave{a}$ une pression extérieure constante, et tordu par des forces appliquées aux extrémités, que l'on suppose infiniment éloignées; un cylindre creux pressé en dehors et en dedans avec des forces différentes; une sphère pleine, dont tous les points s'attirent en raison inverse du quarré des distances; enfin une sphère creuse soumise en dedans et en dehors ì des pressions inégales. Les solutions de ces questions dans lesquelles les déplacemens des points sont donnés par des expressions très simples, et qui se présentent en quelque sorte d'elles-mêmes, offrent les moyens de déterminer de plusieurs manières, d'après les résultats des expériences connues, la valeur d'une constante qui entre dans toutes les formules, et qui peut réprésenter l'intensité de la résistance que les corps de chaque espèce opposent au changement de figure. Ces solutions conduisent à diverses conséquences remarquables. On trouve, par exemple, que lorsque la pression intérieure qui a lieu dans un cylindre creux (qui est supposé d'une longueur infinie) dépasse une certaine limite, il n'est pas possible, quelle que soit l'épaisseur donné à ce cylindre, de le rendre capable de résister à la rupture. Dans le cas d'une sphère pleine dont les points s'attirent en raison inverse du quarré des distances, on trouve qu'au centre la pression intérieure est égale au poids qu'auroit à la surface de la sphère une colonne de la matière dont cette sphère est formée, la longueur de cette colonne étant les $\frac{11}{3} \frac{1}{6}$ du rayon. A une petite profondeur an dessous de la surface, la pression dans le sens du plan tangent est égale au poids d'une semblable colonne dont la hauteur seroit les $\frac{2}{T_{5}}$. du rayon de la sphère. Les parties d'un semblable globe voisines de la surface sont dono comprimées latéralement avec une force extrêmement grande comparativement au poids de la colonne de matière dont elles sont chargées dans le sens du rayon.

MM. Lamé et Clapeyron ont reservé pour le dernier chapitre de leur mémoire plusieurs questions dont la solution comporte une ana- 
lyse d'un ordre plus élevé. Ils s'occupent en premier lieu d'un corps cylindrique pesant, d'une longueur infinie, aux points de la surface duquel sont appliquées des forces distribuées d'une manière arbitraire; puis d'un espace solide d'une étendue indéfinie terminée par un plan ou par deux plans parallèles, en supposant également des forces quelconques appliquées aux points des plans qui forment la surface des corps. La première de ces recherches donne une solution générale des problèmes de la tension et de la torsion d'un corps cylindrique. La seconde conduit à une relation remarquable, et fort simple, entre la condensation et la dilatation qui a lieu près de la surface d'un corps solide, et la pression ou tension appliquée à cette surface, et qui est la cause de l'effet dont il s'agit. On voit reparaître dans ces recherches les formes analytiques au moyen desquelles les questions les plus difficiles de la théorie de la chaleur ont été résolues, et qui semblent destinées désormais à donner aux géomètres les expressions des loix mathématiques des phénomènes naturels les plus importants et les plus divers.

La théorie qui est l'objet de ce mémoire est nouvelle. Cette théorie a depuis quelque tems attiré l'attention de plusieurs géomètres, qui ont présenté sur ce sujet des notions qui ne s'accordent pas entièrement entre elles. Quant aux principes adoptés par les auteurs l'opinion de l'un de vos commissaires, au moins, ne peut être douteuse à cet égard, puisque ces principes ne diffèrent point de ceux qu'il avoit établis depuis longtems dans un écrit,' dont il a été question ci dessus. Cependant, comme le tems et lassentiment général sont nécessaires pour fixer entièrement les idées sur des objets de cette nature, il ne conviendroit pas de proposer ici à lacadémie de prononcer sur ce point un jugement positif. Mais, nónobstant cette reserve, nous n'hésiterons pas à reconnaitre un mérite distingué dans le travail de MM. Lamé et Clapeyron, et à demander que ce travail soit approuvé par l'académie, et imprimé dans le recueil des savans étrangers. Signé à la minute: Poins ot, Navier, rapporteur.

L'Académie adopte les conclusions de ce rapport.

Certifié conforme:

Le sécrétaire perpétuel pour les sciences mathématiques.

$B^{\text {on. }}$. Fourier. 


\section{Mémoire sur l'équilibre intérieur des corps solides.}

\section{Introduction.}

\section{1.}

Les géomètres qui ont étudié la théorie de l'équilibre des corps solides, se sont bornés jusqu'ici à la recherche des relations qui doivent exister entre les forces qui leur sont appliquées, pour qu'elles se contrebalancent exactement. La nature de ces relations est indépendante de la constitution intime du corps; elles résultent de ce que la distance relative des points d'application est supposée invariable.

Mais cette manière d'envisager la question, laisse dans une ignorance complette sur la loi suivant laquelle se transmet, d'un point à l'autre d'un corps solide, l'influence réciproque en vertu de laquelle l'action de l'une des forces est détruite par celle de toutes les autres. L'étude de ce phénomène est pourtant d'une. grande importance, puisque nous voyons que, lorsque les forces qui se font équilibre, acquièrent un degré suffisant d'intensité, le corps solide, après avoir changé de forme d'une manière plus ou moins sensible, finit par se briser.

Les ingénieurs, dans la pratique de leur art, devant proportionner la force de leurs constructions, aux efforts qu'elles doivent supporter, ont sans cesse à s'occuper de considérations de ce genre; aussi ont elles fixé depuis longtems l'attention des géomètres, et la science s'est successivement enrichi de formules importantes sur la résistance des solides; mais toutes celles qui sont parvenues à notre connaissance, fondées sur des hypothèses plus ou moins gratuites, et présentées comme l'expression empyrique des expériences faites par leurs auteurs, occuperaient dans une théorie complette de l'équilibre des corps solides, la place qu'on pourroit assigner aux formules usitées sur le jaugeage des eaux courantes, parmi les loix géné rales de l'hydrostatique, c'est-ì-dire qu'elles seront longtems utiles aux gens de l'art mais qu'une théorie rigoureuse doit leur servir de base.

\section{2.}

Un corps solide peut être considéré comme le lieu géométrique d'un nombre infini de points materiels, qui se distinguent du reste de l'espace par plusieurs propriétés, parmi lesquelles nous citerons celles qui ont trait aux phénomènes mécaniques dont nous recherchons la loi. 
Lorsqu'un corps solide est al l'état de repos, les points matériels qui le composent, sont sollicités par des forces ou nulles, ou qui se font équilibre; mais lorsqu'on exerce une pression ì sa surface, celle-ci entre en mouvement, l'ébranlement se communique aux molécules intérieurs, le corps solide se déforme légèrement, et se constitue bientôt dans un nouvel état d'équilibre. Ce phénomène, sensible dans certains corps, exige des instruments très délicats pour être reconnu dans d'autres, mais il existe pour tous.

Les points matériels placés ì la surface, et qui reçoivent laction immédiate de la pression, transmettent cette pression aux molécules intérieures du corps solide, et éprouvent de leur part une pression égale, qui maintient l'équilibre; ces nouvelles molécules exercent sur des molécules, placées à une plus grande distance de la surface, une action analogue à celle que les molécules de la surface exercent sur elles. Ainsi se propage, suivant une loi inconnue, la pression exerçée ì la surface, jusqu'à ce qu'elle soit détruite par un obstacle contre lequel s'appuie le corps solide.

Si la pression extérieure cesse, tout rentre dans l'état primitif, et les pressions intérieures cessent en même tems.

3.

Soit, par exemple, un corps cylindrique, aux deux bases duquel on applique des tractions égales et opposées, il s'alonge légèrement, et l'équilibre se rétablit ensuite. La traction exercée aux extrémités s'est propagée dans l'intérieur du cylindre, d'une extrémité à lautre: en effet, si l'on imagine une section perpendiculaire aux arêtes, il est nécessaire, pour le nouvel état de l'équilibre, que la partie du corps placée d'un côté de la section, attire celle qui est placée de l'autre côté, et soit attirée par elle par une force égale ì la traction exercée aux extrémités.

Si celle ci étoit remplacée par une compression, le cylindre, au lieu de s'alonger, se raccourcirait, et la partie du corps placée d'un des côtés de la section, exercerait sur lautre, et éprouverait de sa part, une force répulsive égale à la pression qui s'exerce sur les deux extrémités.

Enfin, si l'on fait cesser les tractions ou les pressions extérieures, les attractions ou répulsions intérieures cessent également, et le cylindre reprend sa forme primitive.

\section{4.}

Les changemens de forme d'un corps solide, c'est-à-dire les variations dans les distances respectives des points matériels qui le compo- 


\section{19. Lamé et Clapeyron, md́m. sur C'́quililure int. des corps sol. homog.}

sent, sont donc toujours accompagnés du développement de forees attractives ou répulsives, entre les parties qui le composent; elles naissent, croissent et s'annullent ensemble; ces variations et ces forces sont donc dans une dépendance mutuelle.

Or les propriétés d'un corps solide, ne devant dépendre que de celles des points matériels qui le composent, eux seuls peuvent être considérés comme les foyers d'où émanent les forces dont nous venons de parler; se rappelant en outre que les tractions, exercées sur les extrémités d'un cylindre, donnent naissance à une force attractive dans le sens où les points matériels s'éloignent les uns des autres; et qu'au contraire, une compression donne lieu à une force répulsive, dans le sens où ces points se rapprochent, on est conduit rigoureusement au résultat important dont l'énoncé va suivre, et qui est le fondement de notre théorie.

\section{5.}

Un corps solide en repos, homogène dans toutes ces parties, ì la surface duquel ne s'exerce aucune pression, et dont les molécules ne sont sollicitées par aucune force accélératrice, est le lien d'un nombre infini de points matériels, équidistants, infiniment rapprochés, mais qui ne se touchent pas, et qui jouissent les uns à l'égard des autres de la propriété suivante:

Si en vertu d'une pression extérieure ou d'une force accélératrice qui vient à naître tout-à-coup, deux points pris au hazard se rapprochent ou s'éloignent l'un de l'autre, il en résulte entre ces deux molécules une action ou force répulsive dans le premier cas, et attractive dans le second, qui est une fonction de la distance primitive des deux points et de l'écartement, c'est-ì-dire de la quantité dont ils se sont rapprochés ou éloignés.

Cette fonction pour un même corps est nulle, quelle que soit la distance, lorsque l'écartement est nul; elle décroit très rapidement, quel que soit l'écartement, quand la distance augmente, en sorte que cette fonction Jevient insensible, dès que la distance acquiert une valeur sensible, car toute adhésion cesse entre deux parties d'un même corps séparées l'un de l'autre par une distance appréciable.

Selon que cette fonction variera plus ou moins rapidement avec l'écartement, une même prèssion produira un changement de forme, moins sensible dans le premier cas, et plus sensible dans le second; le premier 
est celui des corps 'rigides, tels que les pierres, les métaux; le second est celui des corps élastiques tels que le caoutchouk.

\section{6.}

La théorie qui va suivre s'applique au cas où le changement de forme, résultant de l'action des pressions extérieures, ou des forces aocélératrices, est très petit; soit que ces pressions ou ces forces soient ellesmêmes très petites, soit que le corps que l'on considère ait une grande rigidité. Alors la fonction de l'écartement et de la distance primitive, qui représente lattraction ou la répulsion, se réduit au produit de la première puissance de lécartement, multipliée par une fonction de la distance primitive, qui, ainsi que nous l'arons remarqué, est insensible dès que la distance acquiert une valeur appréciable.

\section{Première section. Equations différentielles.}

\section{7.}

Soient $x, y, z$ les coordonnées orthogonales du lieu occupé par le centre de gravité d'une molécule quelconque $M$, située dans l'intérieur d'un corps solide homogène; si des forces accélératrices ou des pressions, variables d'une molécule à une autre, viennent à troubler l'homogénéité de ce corps, chaque molécule $M$ s'éloignera de sa première position, en décrivant un espace dont les projections $u, v, w$ seront variables d'une molécule ì-l'autre. Le problème général dont on s'occupe dans ce mémoire, consiste à déterminer les fonctions $u, v, w$ de $x, y, z$, lorsque les circonstances qui ont troublé l'homogénéité sont données, et que le corps soit assez peu déformé, pour que l'on puisse considérer, dans toute son étendue, $u, v, w$, comme très petits par rapport aux dimensions de ce corps.

8.

En exprimant qu'une molécule quelconque $M$, dans sa nouvelle position, est en équilibre, c'est-à-dire que toutes les forces attractives ou répulsives, qui naissent du changement de position de cette molécule $M$, relativement à toutes les autres molécules du corps, font équilibre à la force accélératrice, ayant pour composantes orthogonales $X_{1}, Y_{3}, Z_{1}$, et qui agit sur cette même molécule, on est conduit aux trois équations différentielles: 
154 19. Lamé et Clapeyron, mém. sur l'équilibre int. des corps sol. homoz.

$$
\begin{aligned}
& A\left(\frac{\partial^{2} u}{\partial x^{2}}+\frac{\partial^{2} u}{\partial y^{2}}+\frac{\partial^{2} u}{\partial z^{2}}+2 \frac{\partial\left(\frac{\partial u}{\partial x}+\frac{\partial u}{\partial y}+\frac{\partial w}{\partial z}\right)}{\partial x}\right)+X_{1}=0 \\
& A\left(\frac{\partial^{2} v}{\partial x^{2}}+\frac{\partial^{2} v}{\partial y^{2}}+\frac{\partial^{2} v}{\partial z^{2}}+2 \frac{\partial\left(\frac{\partial u}{\partial x}+\frac{\partial v}{\partial y}+\frac{\partial w}{\partial z}\right)}{\partial y}\right)+Y_{2}=0, \\
& A\left(\frac{\partial^{2} w}{\partial x^{2}}+\frac{\partial^{2} w}{\partial y^{2}}+\frac{\partial^{2} w}{\partial z^{2}}+2 \frac{\partial\left(\frac{\partial u}{\partial x}+\frac{\partial v}{\partial y}+\frac{\partial w}{\partial z}\right)}{\partial z}\right)+Z_{1}=0,
\end{aligned}
$$

$A$ étant un coëfficient constant pour un même corps.

Telles sont les équations générales du problème proposé. (Voyez pour leur démonstration la note première (\$. 15. 16. 17.) à la fin de cette première section.)

\section{9.}

La densité du corps proposé augmente ou diminue en chacun de ses points, lorsqu'il passe de l'état d'équilibre homogène au nouvel état d'équilibre que nous considérons. Or si l'on désigne par $\theta$ le rapport de l'accroissement d'un volume infiniment petit $(\partial x \partial y \partial z)$, occupé par un certain nombre de molécules, à ce volume lui même; cette quantité $\theta$, qu'on peut appeler la dilatation, sera variable d'un point à un autre; de plus elle sera liée aux fonctions $u, v, w$ par l'équation:

$$
\theta=\frac{\partial u}{\partial x}+\frac{\partial v}{\partial y}+\frac{\partial w}{\partial z}
$$

(voyez \$: 18. la note seconde). Les parties du corps où cette fonction $\theta$ sera positive se seront dilatées; celles pour lesquelles $\theta$ sera négative se seront contractées; enfin le lieu de tous les points où la densité du corps n'aura pas changé ou sera restée la même que dans le cas de l'homogénéité, sera représenté par l'équation

$$
\frac{\partial u}{\partial x}+\frac{\partial v}{\partial y}+\frac{\partial w}{\partial z}=0 \text {. }
$$

10.

Les équations (1.) pouvant s'écrire ainsi :

$$
\begin{aligned}
& 3 \cdot \frac{\partial \theta}{\partial x}+\frac{\partial}{\partial y}\left(\frac{\partial u}{\partial y}-\frac{\partial v}{\partial x}\right)-\frac{\partial}{\partial z}\left(\frac{\partial w}{\partial x}-\frac{\partial u}{\partial z}\right)+\frac{X_{\mathrm{I}}}{\mathcal{A}}=0, \\
& 3 \cdot \frac{\partial \theta}{\partial y}-\frac{\partial}{\partial x}\left(\frac{\partial u}{\partial y}-\frac{\partial v}{\partial x}\right)+\frac{\partial}{\partial z}\left(\frac{\partial v}{\partial z}-\frac{\partial w}{\partial y}\right)+\frac{Y_{\mathrm{z}}}{\mathcal{A}}=0, \\
& 3 \cdot \frac{\partial \theta}{\partial z}+\frac{\partial}{\partial x}\left(\frac{\partial w}{\partial x}-\frac{\partial u}{\partial z}\right)-\frac{\partial}{\partial y}\left(\frac{\partial v}{\partial z}-\frac{\partial w}{\partial y}\right)+\frac{Z_{\mathrm{x}}}{\boldsymbol{A}}=0
\end{aligned}
$$

on obtient en les différentiant, la première par rapport à $x$, la seconde 
19. Lamé et Clapeyron, mém. sur l'équilibre int. des corps sol. homog.

par rapport ì $y$, la troisième par rapport ì $z$, et les ajoutant:

$$
\frac{\partial^{2} \theta}{\partial x^{2}}+\frac{\partial^{2} \theta}{\partial y^{2}}+\frac{\partial^{2} \theta}{\partial z^{2}}+\frac{1}{3 A}\left(\frac{\partial X_{x}}{\partial x}+\frac{\partial Y_{x}}{\partial y}+\frac{\partial Z_{x}}{\partial z}\right)=0 \text {. }
$$

Cette équation pourra servir à déterminer directement $\theta$, ou la loi de la dilatation, lorsque les composantes $X_{1}, Y_{1}, Z_{1}$ seront données en $x, y$,

Lorsque la force accélératrice agissant sur chaque molécule sera constante dans toute l'étendue du corps, et $y$ aura constamment la même direction, ce qui comprend le cas d'un corps pesant, situé à la surface de la terre, et peu étendu relativement au rayon terrestre, l'équation précédente se réduit à:

$$
\frac{\partial^{2} \theta}{\partial x^{2}}+\frac{\partial^{2} \theta}{\partial y^{2}}+\frac{\partial^{2} \theta}{\partial z^{2}}=0
$$

On conclût de lì que les dilatations ou contractions qu'un corps solide, homogène et pesant, éprouve intérieurement, lorsque sa surface est soumise à des pressions quelconques, mais non assez fortes pour le déformer d'une manière très sensible, satisfont à l'équation différentielle qui lie les températures permanentes des différents points d'un corps solide, exposé ì des foyers constants de chaleur, ou même les dilatations ou contractions qui doivent résulter de ces témpératures.

11.

Pour intégrer généralement les équations du \$. 8., il convient de chercher d'abord l'intégrale générale $\theta$ (\$. 10.), et de la substituer dans ces équations, mises sous la forme:

$$
\begin{aligned}
& \frac{\partial^{2} u}{\partial x^{2}}+\frac{\partial^{2} u}{\partial y^{2}}+\frac{\partial^{2} u}{\partial z^{2}}+2 \cdot \frac{\partial \theta}{\partial x}+\frac{X_{I}}{A}=0 \\
& \frac{\partial^{2} v}{\partial x^{2}}+\frac{\partial^{2} v}{\partial y^{2}}+\frac{\partial^{2} v}{\partial z^{2}}+2 \cdot \frac{\partial \theta}{\partial y}+\frac{Y_{I}}{A}=0 \\
& \frac{\partial^{2} w}{\partial x^{2}}+\frac{\partial^{2} w}{\partial y^{2}}+\frac{\partial^{2} w}{\partial z^{2}}+2 \cdot \frac{\partial \theta}{\partial z}+\frac{Z_{x}}{A}=0
\end{aligned}
$$

on intégrera ensuite généralement ces équations différentielles, et il ne restera plus qu'ì exprimer que les valeurs générales de $u, v, w, \theta$, satisfont à l'équation:

$$
\theta=\frac{\partial u}{\partial x}+\frac{\partial v}{\partial y}+\frac{\partial w}{\partial z}
$$

Ces intégrations n'offrent par elles mêmes aucune difficulté nouvelle; mais les fonctions arbitraires qui entreront dans les intégrales générales obtenues, doivent être déterminées d'après les conditions données du nouvel état d'équilibre, et cette détermination exige des recherches particuliè- 
156 19. Lamé et Clapeyron, mém. sur Téquilibre int. des corps sol. homog.

res. Pour la faciliter il convient d'envisager la question sous un autre point de vue, et de démontrer les équations du §. 8. d'une autre manière.

12.

Imaginons un plan $P$, parallèle au plan coordonné des $y \approx$, situé i une distance $x$ de ce dernier, et coupant le corps solide proposé. Considérons un cylindre très délié; situé en deça du plan $P$, ayant une base très petite $w$ sur ce plan, et ses arêtes parallèles aux $x$; les molécules du corps situées au delà du plan $P$, ayant changé de position relativement à toutes les molécules situées en deça dans l'intérieur du cylindre à base $w$, il naîtra de ces changemens des forces attractives ou répulsives, qui tendront à déplacer le cylindre; si l'on désigne par $w X, w Y, w Z$ les composantes de la résultante de ces actions, on trouve, en se fondant sur les principes précédemment développés :

$$
X=A\left(3 \frac{\partial w}{\partial x}+\frac{\partial v}{\partial y}+\frac{\partial w}{\partial z}\right), \quad Y=A\left(\frac{\partial v}{\partial x}+\frac{\partial u}{\partial y}\right), Z=A\left(\frac{\partial w}{\partial x}+\frac{\partial u}{\partial z}\right)
$$

$A$ étant toujours un coëfficient numérique dépendant de la nature du corps. Si les compasantes $w X, w Y, w Z$ sont positives, c'est que le cylindre tend à être entrainé dans le sens des $x, y$, ou $z$ positifs; si elles sont négatives au contraire, c'est que ce cylindre tend à marcher du côté des $x, y, z$ négatifs. On pourra dire que dans le premier cas la portion du corps située au delà du plan $\boldsymbol{P}$ attire la base $w$, et que dans le second elle Ia presse ou la repousse. (Voyez la note 3. (\$. 19. et 20.) pour la démonstration des équations précédentes.)

\section{3.}

Si le plan $P$ est parallèle au plan des $z x$, et situé i une distance $\boldsymbol{y}$ de lorigine, que le cylindre d̀ base $w$ ait ses arêtes parallèles aux $y$, et que $w X^{\prime}, w Y^{\prime}$, $w Z^{\prime}$ représentent les composantes de la résultante des actions exercées sur les molécules intérieures à ce cylindre, par toutes celles situées au delì du plan $P$, on trouve pareillement:

$$
X^{\prime}=A\left(\frac{\partial u}{\partial y}+\frac{\partial v}{\partial x}\right), \quad Y^{\prime}=A\left(\frac{\partial u}{\partial x}+3 \frac{\partial v}{\partial y}+\frac{\partial w}{\partial z}\right), \quad Z^{\prime}=A\left(\frac{\partial w}{\partial y}+\frac{\partial v}{\partial z}\right) .
$$

Enfin si le plan $P$ est parallèle au plan de $x y$, et situé ì une distance $z$ de l'origine, que le cylindre ait ses arêtes parallèles aux $z$, et que $w X^{\prime \prime}, w Y^{\prime \prime}, w Z^{\prime \prime}$ représentent les composantes de la résultante des actions exercées sur le cylindre par les molécules du corps situées au dessus du plan $P$, on troure: 


$$
X^{\prime \prime}=A\left(\frac{\partial u}{\partial z}+\frac{\partial w}{\partial x}\right), \quad V^{\prime \prime}=A\left(\frac{\partial v}{\partial z}+\frac{\partial w}{\partial y}\right), Z^{\prime \prime}=A\left(\frac{\partial u}{\partial x}+\frac{\partial v}{\partial y}+3 \frac{\partial w}{\partial z}\right) .
$$

On trouverait les mêmes valeurs, mais affectées de signes contraires, si, dans ces trois cas différens, on supposait que le cylindre, s'appuyant toujours sur la même base $w$, fut situé au dessus du plan $P$, et que l'on cherchât les actions exercées sur ce cylindre, par les molécules situées au dessous de ce plan.

\section{4.}

Les équations des paragraphes précédens conduisent aux équations différentielles du \$. 8. Pour le faire voir, considérons, dans l'intérieur du corps proposé, un parallelepipède $h k l$, dont les cotés $h, k, l$ soient parallèles aux axes coordonnés et très petits; les molécules intérieures à ce parallèlepipède, ayant changé de position relativement à toutes les autres molécules du corps, ces changemens ont fait naître des attractions ou des répulsions, tendant à faire mouvoir le parallèlepipède, et faisant équilibre aux forces accélératrices, étrangères au corps, qui peuvent lui être appliquées; proposons nous de chercher les équations qui expriment que cet équilibre a lieu.

Représentons par $x, y$, $z$ les coordonnées du sommet du prisme $h k l$, le plus voision de lorigine, et soient $x^{\prime}, y^{\prime}, z^{\prime}$ les coordonnées courantes; la portion du corps, inférieure au plan $x^{\prime}=x$, exercera sur la base $k l$ du prisme, située dans ce plan, une traction ou pression ayant pour composantes $k l x, k l y, k l z$; la portion du corps supérieuré au plan $x^{\prime}=x+h$ exercera sur la surface $k l$ du prisme située dans ce plan, une traction ou pression ayant pour composante $\left(X+\frac{\partial X}{\partial x} h\right) k l,\left(Y+\frac{\partial Y}{\partial x} h\right) k l$, $\left(Z+\frac{\partial Z}{\partial x} h\right) k l$; ainsi la résultante des actions exercées sur les deux bases $k l$ du parallèlepipède $h k l$, aura pour composantes:

$$
\frac{\partial X}{\partial x} h k l, \frac{\partial Y}{\partial x} h k l, \frac{\partial Z}{\partial x} h k l ;
$$

on trouvera de la même manière que les actions exercées sur les deux faces $h l$, ont pour composantes:

$$
\frac{\partial X^{\prime}}{\partial y} h k l, \frac{\partial Y^{\prime}}{\partial y} h k l, \frac{\partial Z^{\prime}}{\partial z} h k l ;
$$

et qu'enfin les actions exercées sur les deux faces $h k$ conduisent aux composantes:

$$
\frac{\partial X^{\prime \prime}}{\partial z} h k l, \frac{\partial Y^{\prime \prime}}{\partial \gamma} h k l, \frac{\partial Z^{\prime \prime}}{\partial z} h k l \text {. }
$$


158 19. Lamć et Clapeyron, mém. sur l'équilibre int. des corps sol. homog.

Toutes ces tractions ou pressions font équilibre aux résultantes des forces accélératrices agissant sur le parallèlepipède $h k l$, et qui sont:

$$
X_{1} h k l, Y_{2} h k l, Z_{2} h k l \text {; }
$$

on a donc nécessairement les trois équations:

$$
\begin{aligned}
& \frac{\partial X}{\partial x}+\frac{\partial X^{\prime}}{\partial y}+\frac{\partial X^{\prime \prime}}{\partial z}+X_{1}=0 \\
& \frac{\partial Y}{\partial x}+\frac{\partial Y^{\prime}}{\partial y}+\frac{\partial Y^{\prime \prime}}{\partial z}+Y_{1}=0 \\
& \frac{\partial Z}{\partial x}+\frac{\partial Z^{\prime}}{\partial y}+\frac{\partial Z^{\prime \prime}}{\partial z}+Z_{1}=0
\end{aligned}
$$

Si l'on substitue dans ces dernières équations les valeurs de $X, Y, Z$, $X^{\prime}, Y^{\prime}, Z^{\prime}, X^{\prime \prime}, Y^{\prime \prime}, Z^{\prime \prime}$, trouvées précédemment (\$. 12. et 13.), elles deviennent identiques avec les équations du \$. 8.

Note première. Démonstration des équations différentielles.

15.

Soient $M$ et $M^{\prime}$ deux molécules très voisines l'une de l'autre, d'un corps solide homogène, occupant, avant toute espèce de dérangement de la part de forces accélératrices ou de pressions extérieures, les points dont les coordonnées, rapportées à trois axes rectangulaires, sont $x, y, z, x^{\prime}$, $y^{\prime}, z^{\prime}$; soit 5 leur distance. Si des forces accélératrices ou des pressions extérieures viennent à agir sur le corps, chacune des deux molécules $\boldsymbol{M}$ et $M^{i}$ décrira des espaces très petits, dont les projections sur les trois axes seront $u, v, w$ pour le premier, $u^{\prime}, v^{\prime}, w^{\prime}$ pour le second. Après ce mouvement, et lorsque le nouvel état d'équilibre sera rétabli, les deux molécules $M$ et $M^{\prime}$ occuperont des points $m$ et $m^{\prime}$; appelant alors $\Delta z$ la quantité très petite dont elles se seront écartées, il naîtra entr'elles une force attractive égale à la somme de leurs masses, multipliée par $\Delta z$, et par une fonction de la distance primitive $\zeta$, ou $F(\zeta)$, assujétie à devenir insensible, dès que $\zeta$ acquiert une valeur appréciable.

La distance $M M^{\prime}$ ou $\zeta$, avoit pour projections sur les trois axes $x^{\prime}-x, y^{\prime}-y, z^{\prime}-z$; la distance $m m^{\prime}$ ou $\zeta+\Delta \zeta$ aura pour projections: $x^{\prime}-x+u^{\prime}-u, y^{\prime}-y+v^{\prime}-v, z^{\prime}-z+w^{\prime}-w$. Si par le point $m$ on mène une droite $m \mu^{\prime}$, égale et parallèle à $M M^{\prime}$, la distance $\mu^{\prime} m^{\prime}$ aura évidemment pour projections $u^{\prime}-u, v^{\prime}-v, w^{\prime}-w$; or en vertu de la petitesse des mouvements $M m, M^{\prime} m^{\prime}$, relativement i $x^{\prime}-x, y^{\prime}-y, z^{\prime}-z$, la ligne $m m^{\prime}$, peut être considérée comme faisant un angle infiniment pe- 
19. Lamé et Clapeyron, mém. sur C'́quilibre int. des corps sol. homog. 159 tit avec $M M$ ou $m \mu^{\prime}$, car les cosinus $\frac{x^{\prime}-x+u^{\prime}-u}{\zeta+\Delta \zeta}, \frac{y^{\prime}-y+v^{\prime}-v}{\zeta+\Delta \zeta}$, $\frac{z^{\prime}-z+w^{\prime}-w}{\zeta+\Delta \zeta}$, des angles formés avec les axes par la direction $m m^{\prime}$, diffèrent très peu des cosinus $\frac{x^{\prime}-x}{\zeta}, \frac{y^{\prime}-y}{\zeta}, \frac{z^{\prime}-z}{\zeta}$ des angles correspondans à la direction $M M^{\prime}$ ou $m \mu^{\prime}$; il suit delà que la différence des distances $m \mu^{\prime}$ ou $\zeta$, et $m m^{\prime}$ ou $\zeta+\Delta \zeta$ peut être considérée comme égale à la projection de $\mu^{\prime} m^{\prime}$ sur $m m^{\prime}$ ou sur $M M^{\prime}$, puisque ces deux lignes peuvent être considérées comme parallèles. On aura donc $\Delta \zeta$, en multipliant respectivement les projections $u^{\prime}-u, v^{\prime}-v, w^{\prime}-w$, de $\mu^{\prime} m^{\prime}$, par les cosinus des angles que $M M^{\prime}$ fait avec les axes; ainsi on peut poser:

$$
\Delta \zeta=\left(u^{\prime}-u\right) \frac{x^{\prime}-x}{\zeta}+\left(v^{\prime}-v\right) \frac{y^{\prime}-y}{\zeta}+\left(w^{\prime}-w\right) \frac{z^{\prime}-z}{\zeta} .
$$

16.

Soient $x^{\prime}-x=h, y^{\prime}-y=k, z^{\prime}-z=l ; h, k, l$ seront des quantités insensibles, puisque la distance $M^{\prime} M$ est très petite; or $u^{\prime}, v^{\prime}, w^{\prime}$ n'étant autre chose que ce que deviennent $u, v, w$, lorsqu'on substitue dans ces fonctions $x+h, r+k, z+l$, au lieu de $x, y$, on aura, daprès le théorème de Taylor:

$$
\begin{aligned}
u^{\prime}-u= & \frac{\partial u}{\partial x} h+\frac{\partial u}{\partial y} k+\frac{\partial u}{\partial z} l+\frac{\partial^{2} u}{\partial x^{2}} \cdot \frac{h^{2}}{2}+\frac{\partial^{2} u}{\partial^{2} y} \cdot \frac{k^{2}}{2}+\frac{\partial^{2} u}{\partial^{2} z^{2}} \cdot \frac{l^{2}}{2} \\
& +\frac{\partial^{2} u}{\partial x \partial y} h k+\frac{\partial^{2} u}{\partial y \partial z} k l+\frac{\partial^{2} u}{\partial z \partial x} l h+\text { etc. },
\end{aligned}
$$

et de la même manière:

$$
\begin{aligned}
& v^{\prime}-v=\frac{\partial v}{\partial x} h+\frac{\partial v}{\partial y} k+\frac{\partial v}{\partial z} l+\text { etc. }, \\
& w^{\prime}-w=\frac{\partial w}{\partial x} h+\frac{\partial w}{\partial y} k+\frac{\partial w}{\partial z} l+\text { etc. }
\end{aligned}
$$

Ces valeurs substituées dans $\Delta \zeta$, donneront cette quantité en fonction des accroissemens variables $h, k, l$ et des coëfficiens différéntiels de $u, v, w$, qui pourront être regardés comme constants lorsque $h, k, l$ varieront.

La force qui agit sur la molécule $m$ pour la ramener vers $m^{\prime}$, a donc pour composantes suivant les trois axes:

$$
\begin{aligned}
& {\left[\left(u^{\prime}-u\right) \frac{h}{\zeta}+\left(v^{\prime}-v\right) \frac{k}{\zeta}+\left(v^{\prime}-w\right) \frac{l}{\zeta}\right] F(\zeta) \frac{h}{\zeta},} \\
& {\left[\left(u^{\prime}-u\right) \frac{h}{\zeta}+\left(v^{\prime}-v\right) \frac{k}{\zeta}+\left(w^{\prime}-w\right) \frac{l}{\zeta}\right] F(\zeta) \frac{k}{\zeta},} \\
& {\left[\left(u^{\prime}-u\right) \frac{h}{\zeta}+\left(v^{\prime}-v\right) \frac{k}{\zeta}+\left(w^{\prime}-w\right) \frac{l}{\zeta}\right] F(\zeta) \frac{l}{\zeta},}
\end{aligned}
$$


Pour simplifier nous pourrons supposer que les masses des molécules $M$ et $M^{\prime}$ entrent comme facteurs dans $F(\zeta)$, puisque ne considérant que des corps homogènes, nous n'aurons jamais besoin de dégager ce facteur constant.

La force dont nous venons d'évaluer les projections n'est pas la seule qui agisse sur la molécule $M$, suivant la direction $M M^{\prime}$ et à distance ¿; si l'on considère la molécule $M^{\prime \prime}$ située sur $M^{\prime} M$, à une distance $M^{\prime \prime} M=\zeta$ de l'autre coté du point $M$, la distance $M " M$ ayant changé, et étant devenue $\zeta+\Delta \zeta$, il en résultera une force nouvelle, qui tendra à faire marcher $M$ vers $M^{\prime \prime}$, et qui aura pour composantes:

$$
\begin{aligned}
& -\left[\left(u-u^{\prime \prime}\right) \frac{h}{\zeta}+\left(v-v^{\prime \prime}\right) \frac{k}{\zeta}+\left(w-w^{\prime \prime}\right) \frac{l}{\zeta}\right] F(\zeta) \frac{h}{\zeta}, \\
& -\left[\left(u-u^{\prime \prime}\right) \frac{h}{\zeta}+\left(v-v^{\prime \prime}\right) \frac{k}{\zeta}+\left(w-w^{\prime \prime}\right) \frac{l}{\zeta}\right] F(\zeta) \frac{k}{\zeta}, \\
& -\left[\left(u-u^{\prime \prime}\right) \frac{h}{\zeta}+\left(v-v^{\prime \prime}\right) \frac{k}{\zeta}+\left(w-w^{\prime \prime}\right) \frac{l}{\zeta}\right] F(\zeta) \frac{l}{\zeta},
\end{aligned}
$$

$u^{\prime \prime}, v^{\prime \prime}, w^{\prime \prime}$ étant ce que deviennent, lorsqu'on y change $x, y, z$ en $x-h$, $y-k, z-l$.

En vertu des actions réunies des molécules $M^{\prime}$ et $M^{\prime \prime}$ sur $M$, elle tendra à marcher dans la direction $M M^{\prime}$, en vertu d'une force dont les composantes seront:

$$
\begin{aligned}
& {\left[\left(u^{\prime}+u^{\prime \prime}-2 u\right) \frac{h}{\zeta}+\left(v^{\prime}+v^{\prime \prime}-2 v\right) \frac{k}{\zeta}+\left(w^{\prime}+w^{\prime \prime}-2 w\right) \frac{l}{\zeta}\right] F(\zeta) \frac{h}{\zeta},} \\
& {\left[\left(u^{\prime}+u^{\prime \prime}-2 u\right) \frac{h}{\zeta}+\left(v^{\prime}+v^{\prime \prime}-2 v\right) \frac{k}{\zeta}+\left(w^{\prime}+w^{\prime \prime}-2 w\right) \frac{l}{\zeta}\right] F(\zeta) \frac{k}{\zeta},} \\
& {\left[\left(u^{\prime}+u^{\prime \prime}-2 u\right) \frac{h}{\zeta}+\left(v^{\prime}+v^{\prime \prime}-2 v\right) \frac{k}{\zeta}+\left(w^{\prime}+w^{\prime \prime}-2 w\right) \frac{l}{\zeta}\right] F(\zeta) \frac{l}{\zeta} .}
\end{aligned}
$$

On peut obtenir les valeurs de $\left(u^{\prime \prime}-u\right),\left(v^{\prime \prime}-v\right),\left(w^{\prime \prime}-w\right)$, en changeant les signes de $h, k, l$ dans les valeurs de $u^{\prime}-u, v^{\prime}-v, w^{\prime}-w$, déduites précédemment du théorème de Taylor; c'est ce qui permettra de trouver les valeurs de $u^{\prime}+u^{\prime \prime}-2 u, v^{\prime}+v^{\prime \prime}-2 v, w^{\prime}+w^{\prime \prime}-2 w$, en fonction de $h, k, l ;$ si on substitue ces valeurs dans les expression précédentes, en négligeant les produits de trois dimensions de $h, k, l$ devant ceux de deux dimensions seulement, on aura:

$$
\begin{aligned}
& {\left[\left(\frac{\partial^{2} u}{\partial x^{2}} h^{2}+\frac{\partial^{2} u}{\partial y^{2}} k^{2}+\frac{\partial^{2} u}{\partial z^{2}} l^{2}+2 \frac{\partial^{2} u}{\partial y} \frac{\partial}{\partial z} k l+2 \frac{\partial^{2} u}{\partial z \partial x} l h+2 \frac{\partial^{2} u}{\partial x} h y k\right) \frac{h}{\zeta}\right.} \\
+ & \left(\frac{\partial^{2} v}{\partial x^{2}} h^{2}+\frac{\partial^{2} v}{\partial y^{2}} k^{2}+\frac{\partial^{2} v}{\partial z^{2}} l^{2}+2 \frac{2 \partial^{2} v}{\partial y} k l+2 \frac{\partial^{2} v}{\partial z \partial x} l h+2 \frac{\partial^{2} v}{\partial x} h h k\right) \frac{k}{\zeta} \\
+ & \left.\left(\frac{\partial^{2} w}{\partial x^{2}} h^{2}+\frac{\partial^{2} w}{\partial y^{2}} k^{2}+\frac{\partial^{2} w}{\partial z^{2}} l^{2}+2 \frac{2 \partial^{2} w}{\partial y} k l+2 \frac{\partial^{2} w}{\partial z \partial x} l h+2 \frac{\partial^{2} w}{\partial x} h k\right) \frac{l}{\zeta}\right] F(\zeta) \frac{h}{\zeta},
\end{aligned}
$$


pour la composante suivant l'axe des $x$, de la résultante des actions des molécules $M^{\prime}$ et $M^{\prime \prime}$ sur $M$; les composantes de cette même résultante sur les axes des $y$ et des $z$ seront évidemment égales ì la précédente, respectivement multipliée par $\frac{k}{h}$ et $\frac{l}{h}$.

17.

Soient $\varphi$ langle ì l'horizon de la direction $M M^{\prime}$, et $\psi$ l'angle que le plan vertical, dans lequel elle se trouve, fait avec celui des $z x$, on aura $h=\zeta \cos \varphi \cos \psi, k=\zeta \cos \varphi \sin \psi, l=\zeta \sin \varphi$. Toutes les molécules voisines de $M^{\prime}$, et situées dans l'élément sphérique $\left[\zeta^{2} \cos \varphi \partial \zeta \partial \varphi \partial \psi\right]$, pouvant être considérées comme agissant de la même manière sur la molécule $M$, il suffira de multiplier les composantes, dont l'expression est indiquée à la fin du paragraphe précedent, par cet élément sphérique et d'intégrer chacune d'elles trois fois: l'une par rapport ì $\varphi$ depuis $\varphi=0$ jusqu'ì $\varphi=\frac{\pi}{2}$; la seconde par rapport $\grave{a} \psi$, de $\psi=0$ ì $\psi=2 \pi$; la troisième depuis $\zeta=0$ jusqu'd $\zeta=\infty$, car $F(\zeta)$ devenant nulle, lorsque $\zeta$ a une valeur sensible, on peut prendre ces deux limites pour $\zeta$; on aura alors, par toutes ces intégrations, les composantes de la résultante de toutes les forces qui tendent ì faire marcher la molécule $M$ ou à s'éloigner de sa seconde position d'équilibre.

Si l'on effectue toutes ces intégrations, que l'on représente $\left[\frac{2}{15} \pi \int_{0}^{\infty} \zeta^{4} F(\zeta) \partial \zeta\right]$ par une constante $A$, les composantes de la résultante dont nous venons de parler seront, après toute réduction faite:

$$
\begin{aligned}
& A\left(\frac{\partial^{2} u}{\partial x^{2}}+\frac{\partial^{2} u}{\partial y^{2}}+\frac{\partial^{2} u}{\partial z^{2}}+2 \frac{\partial\left(\frac{\partial u}{\partial x}+\frac{\partial v}{\partial y}+\frac{\partial w}{\partial z}\right)}{\partial x}\right), \\
& A\left(\frac{\partial^{2} v}{\partial x^{2}}+\frac{\partial^{2} v}{\partial y^{2}}+\frac{\partial^{2} v}{\partial z^{2}}+2 \frac{\partial\left(\frac{\partial u}{\partial x}+\frac{\partial v}{\partial y}+\frac{\partial w}{\partial z}\right)}{\partial y}\right), \\
& A\left(\frac{\partial^{2} w}{\partial x^{2}}+\frac{\partial^{2} w}{\partial y^{2}}+\frac{\partial^{2} w}{\partial z^{2}}+2 \frac{\partial\left(\frac{\partial u}{\partial x}+\frac{\partial v}{\partial y}+\frac{\partial w}{\partial z}\right)}{\partial z}\right) .
\end{aligned}
$$

Si en passant du premier au second état d'équilibre, les molécules du corps solide proposé deviennent soumises à l'action de forces accélératrices dont les composantes sont $X_{1}, Y_{2}, Z_{1}$, pour la molécule $M$, son équilibre exigera que lon ait:

Crelle's Journal d. M. VII. Bd. 2. Hft. 
19. Lamé et Clapeyron, mém. sur l'équilibre int. des corps sol. homog.

$$
\begin{aligned}
& \frac{\partial^{2} u}{\partial x^{2}}+\frac{\partial^{2} u}{\partial y^{2}}+\frac{\partial^{2} u}{\partial z^{2}}+2 \cdot \frac{\partial\left(\frac{\partial u}{\partial x}+\frac{\partial v}{\partial y}+\frac{\partial w}{\partial z}\right)}{\partial x}+\frac{X_{\mathrm{x}}}{A}=0, \\
& \frac{\partial^{2} v}{\partial x^{2}}+\frac{\partial^{2} v}{\partial y^{2}}+\frac{\partial^{2} v}{\partial z^{2}}+2 \cdot \frac{\partial\left(\frac{\partial u}{\partial x}+\frac{\partial v}{\partial y}+\frac{\partial w}{\partial z}\right)}{\partial y}+\frac{Y_{\mathrm{x}}}{A}=0, \\
& \frac{\partial^{2} w}{\partial x^{2}}+\frac{\partial^{2} w}{\partial y^{2}}+\frac{\partial^{2} w}{\partial z^{2}}+2 \cdot \frac{\partial\left(\frac{\partial u}{\partial x}+\frac{\partial v}{\partial y}+\frac{\partial w}{\partial z}\right)}{\partial z}+\frac{Z_{\mathrm{x}}}{A}=0 .
\end{aligned}
$$

Dans ces équations, on peut supposer que la masse de la molecu'e $M$, ou la densité du corps homogène proposé, n'entre pas, parcequ'elle aurait dû entrer comme facteur dans $X_{1}, Y_{1}, Z_{1}$ et $A$, et conséquemment disparaître comme facteur commun. La seule quantité qui puisse varier d'un corps à l'autre dans les équations précédentes est $A=\frac{2}{15} \pi \int_{0}^{\infty} \zeta^{4} F(\zeta) \partial \zeta$; on voit que plus cette quantité sera petite, plus $u, v, w$ seront grands; $A$ est une constante qui dépend de l'élasticité du corps.

Note seconde. Expression de la dilatation.

18.

Soient deux molécules $M$ et $M^{\prime}$, situées dans le corps solide homogène, sur une même ligne parallèle ì l'axe des $x$, et très voisines l'une de l'autre, en sorte que la distance $h$, qui les sépare soit insensible; les coordonnées du point $M$ étant $x, y, z$, celles du point $M^{\prime}$ seront $x+h$, $y$, $z$; lors du second état d'équilibre, la molécule $M$ prendra une autre position $m$, et aura pour coordonnées $x+u, y+v, z+w$; la molécule $M^{\prime}$ prendra la position $m^{\prime}$ et aura pour coordonnées $x+h+u+\frac{\partial u}{\partial x} h$, $y+v+\frac{\partial v}{\partial x} h, z+w+\frac{\partial w}{\partial x} h$; mais, d'après les considérations de la note précédente, les lignes $M M^{\prime}, m m^{\prime}$, faisant un angle infiniment petit, $m m^{\prime}$ pourra encore être considérée comme parallèle à l'axe des $x$, et sa longueur sera simplement $h\left(1+\frac{\partial u}{\partial x}\right)$. Si l'on considère un point $M^{\prime \prime}$ situé à une distance insensible $k$ de $M$, sur la parallèle à l'axe des $y$ menée par $M$, on démontrera de la même manière, que du premier au second état d'équilibre, la distance $M M^{\prime \prime}=k$ deviendra $m m^{\prime}=k\left(1+\frac{\partial v}{\partial y}\right)$. Enfin si l'on considère une molécule $M^{\prime \prime \prime}$, située à une distance $l$ de $M$ sur une 
parallèle à l'axe des $z$, la distance $M M^{\prime \prime \prime}=l$ deviendra $m m^{\prime \prime}=l\left(1+\frac{\partial w}{\partial z}\right)$. Il suit delì que le parallèlepipède $h k l$ deviendra lors du second état d'équilibre $h k l\left(1+\frac{\partial u}{\partial x}\right)\left(1+\frac{\partial v}{\partial y}\right)\left(1+\frac{\partial w}{\partial z}\right)$, ou simplement $h k l\left(1+\frac{\partial u}{\partial x}+\frac{\partial v}{\partial y}+\frac{\partial w}{\partial z}\right)$, si on néglige les rectangles des coëfficiens différentiels $\frac{\partial u}{\partial x}, \frac{\partial v}{\partial y}, \frac{\partial w}{\partial z}$, comme disparaissant devant leur somme, puisque $u, v, w$ sont très petits par rapport ì $x, y, z$, ou plutôt $\partial u, \partial v, \partial w$, par rapport à $\partial x, \partial y, \partial z$. Le volume primitif du parallèlepipède rectangle $h k l$, augmente donc, dans le second état d'équilibre de $h k l\left(\frac{\partial u}{\partial x}+\frac{\partial v}{\partial y}+\frac{\partial w}{\partial z}\right)$, ainsi $\frac{\partial u}{\partial x}+\frac{\partial v}{\partial y}+\frac{\partial w}{\partial z}$ est le rapport de l'augmentation de volume de ce parallèlepipède à ce volume, c'est ce qu'on peut appeler la dilatation de ce volume même; soit $\theta$ cette quantité ou cette dilatation, qui sera fonction de $x, y, z$, on aura identiquement:

$$
\theta=\frac{\partial u}{\partial x}+\frac{\partial v}{\partial y}+\frac{\partial w}{\partial z} \text {. }
$$

Note troisième. Démonstration des formules relatives aux pressions intérieures.

19.

Imaginons: par la molécule $M$ un plan $P^{\prime \prime}$ perpendiculaire aux $\zeta$, et conséquemment parallèle au plan des $x y$; sur ce plan une base très petite $w$, comprenant le point $M$, et enfin un cylindre infiniment délié, ayant pour base $w$ et ses arêtes parallèles aux $\zeta$, et dirigées vers l'origine. Proposons nous de trouver la résultante des actions que les molécules du corps, situées au dessus du plan $P^{\prime \prime}$ exercent sur celles, situées dans lintérieur du cylindre infiniment délié dont nous venons de parler, en vertu du dérangement qu'a occasionné le second état d'équilibre.

Une molécule $M^{\prime}$ devenue $m^{\prime}$ exercera sur la molécule $M$ devenue $m$, une action dirigée suivant $M M^{\prime}$ ou $m m^{\prime}$ égale à :

$$
\left[\left(u^{\prime}-u\right) \frac{h}{\zeta}+\left(v^{\prime}-v\right) \frac{k}{\zeta}+\left(w^{\prime}-w\right) \frac{l}{\zeta}\right] F(\zeta),
$$

d'après ce qui a été dit (Note première $\$$.15. et 16.), $\zeta$ étant la distance $M M^{\prime} ; h, k, l$ les projections de cette distance sur les trois axes; $u, v, w$ les projections de $M m ; u^{\prime}, v^{\prime}, w^{\prime}$ celles de $M^{\prime} m^{\prime}$; enfin $F(\zeta)$ une fonction de $\zeta$, qui décroit très rapidement, lorsque $\zeta$ croit, et qui est insensible ou nulle pour des valeurs sensibles de $\zeta$. D'après cela, on pourra poser: 
164 19. Lamé et Cla peyron, mém. sur l'équilibre int. des corps sol. homog̈.

$$
\begin{gathered}
u^{\prime}-u=\frac{\partial u}{\partial x} h+\frac{\partial u}{\partial y} k+\frac{\partial u}{\partial z} l ; \quad v^{\prime}-v=\frac{\partial v}{\partial x} h+\frac{\partial v}{\partial y} k+\frac{\partial v}{\partial z} l ; \\
w^{\prime}-w=\frac{\partial w}{\partial x} l+\frac{\partial w}{\partial y} k+\frac{\partial w}{\partial z} l ;
\end{gathered}
$$

la force dirigée suivant $\boldsymbol{M}^{\prime} \boldsymbol{M}$, aura ainsi pour expression nouvelle: $\left.\left(\frac{\partial u}{\partial x} h+\frac{\partial u}{\partial y} k+\frac{\partial u}{\partial z} l\right) \frac{h}{\zeta}+\left(\frac{\partial v}{\partial x} h+\frac{\partial v}{\partial y} k+\frac{\partial v}{\partial z} l\right) \frac{k}{\zeta}+\left(\frac{\partial w}{\partial x} h+\frac{\partial w}{\partial y} k+\frac{\partial w}{\partial z} l\right) \frac{l}{\zeta}\right] F(\zeta)$. Soit, comme dans la note première: $h=\zeta \cos \varphi \cos \psi, k=\zeta \cos \varphi \sin \psi$, $l=\zeta \sin \varphi$; toutes les molécules situées dans l'espace infiniment petit ou élément sphérique $\left[\zeta^{2} \cos \varphi \partial \varphi \partial \psi \partial \zeta\right]$, dont $M^{\prime}$ fait partie, pourront être considérées comme agissant chacune, sur chacune des molécules de l'élément infiniment petit $w \partial p$ du cylindre proposé qui contient $M$, avec une force de même intensité et de même direction que la force exercée par $M^{\prime}$ sur $M$; ainsi l'action totale de l'élément sphérique $\left[\zeta^{2} \cos \varphi \partial \varphi \partial \psi \partial \zeta\right]$ sur l'élément cylindrique $w \partial p$ sera égale à:

a. $\left[\left(\frac{\partial u}{\partial x} \cos \varphi \cos \psi+\frac{\partial u}{\partial y} \cos \varphi \sin \psi+\frac{\partial u}{\partial z} \sin \varphi\right) \cos \varphi \cos \psi\right.$ $+\left(\frac{\partial v}{\partial x} \cos \varphi \cos \psi+\frac{\partial v}{\partial y} \cos \varphi \sin \psi+\frac{\partial v}{\partial z} \sin \varphi\right) \cos \varphi \sin \psi$ $\left.+\left(\frac{\partial w}{\partial x} \cos \varphi \cos \psi+\frac{\partial w}{\partial y^{\prime}} \cos \varphi \sin \psi+\frac{\partial w}{\partial z} \sin \varphi\right) \sin \varphi\right] \zeta^{3} F(\zeta) \cos \varphi \partial \varphi \partial \psi \partial \partial w \partial p ;$ les composantes de cette action s'obtiendront en multipliant successivement cette expression par $\cos \varphi \cos \psi, \cos \varphi \sin \psi, \sin \varphi$.

20.

Soit $M_{1}$ une molécule du cylindre proposé située à une distance $p$ de la molécule $M$, et dont les coordonnées seront $x, y, z-p$; soit une molécule $M_{a}^{\prime}$, située sur une parallèle aux $\zeta$ menée par $M^{\prime}$, à une distance $p$ au dessous de $M^{\prime}$, ses coordonnées seront: $x+h, y+k$, $z+l-p$; la ligne $M_{1} M_{1}^{\prime}$ sera évidemment parallèle à $M M^{\prime}$, et lui sera égale en longueur. Si l'on désigne par $u_{1}, v_{1}, w_{1}$ les projections de l'élément parcouru par la molécule $M_{1}$, lorsqu'elle passe à la seconde position d'équilibre, par $u_{\mathrm{x}}^{\prime}, v_{\mathrm{x}}^{\prime}, w_{1}^{\prime}$, les mêmes choses pour $M_{\mathrm{x}}^{\prime}$, on aura évidemment ces quantités en substituant dans $u, v, w: x, y, \zeta-p$ au lieu de $x, y, z$, et dans $u^{\prime}, v^{\prime}, w^{\prime}: x+h, y+k, z+l-p$ au lieu de $x+h$, $y+k, z+l$; or comme la molécule $M_{1}^{\prime}$, quoique au dessous de $M^{\prime}$, doit être au dessus du plan $P^{\prime \prime}, p$ doit être plus petit que $\zeta$, et du même ordre de grandeur que $h, k, l$, en sorte que $p^{2}$ devra être négligé devant $p$; on aura donc:

$$
u_{1}=u-\frac{\partial u}{\partial z} p, \quad u_{x}^{\prime}=u+\frac{\partial u}{\partial x} h+\frac{\partial u}{\partial y} k+\frac{\partial w}{\partial z}(l-p)
$$


19. Lamé et Clapeyron, mém. sur l'ćquilibre int. des corps sol. homog. 165 et par suite:

$$
u_{\mathrm{s}}^{\prime}-u_{1}=\frac{\partial u}{\partial x} h+\frac{\partial u}{\partial y} k+\frac{\partial u}{\partial z} l=x^{\prime}-u_{\bullet}
$$

On démontrera de la même manière que $v_{1}^{\prime}-v_{1}=v^{\prime}-v$, que $w_{\mathrm{x}}^{\prime}-w_{1}=w^{\prime}-w$; il suit delì, que l'action d'un élément sphérique, dont $M_{1}^{\prime}$, fera partie, sur un élément cylindrique $w \partial p$, dont $M_{1}$ fera partie, aura identiquement la même expression que celle $\left(a_{0}\right)$ trouvée plus haut; ainsi, en intégrant cette expression ( $\left.\alpha_{0}\right)$ par rapport à $p$, depuis $p=0$ jusqu'ì $p=\zeta \sin \varphi$, on aura la somme des actions dirigées suivant la direction $(\varphi \psi)$ et exercées a la distance $\zeta$, par les molécules situées sur la ligne $M^{\prime} M_{1}^{\prime}$ au dessus du plan $P^{\prime \prime}$, sur les molécules du cylindre infiniment délié proposé. Si l'on intègre ensuite cette expression, successivement multipliée par $\cos \varphi \cos \psi, \cos \varphi \sin \psi, \sin \varphi$, par rapport à $\zeta$, de $\zeta=0$ à $\zeta=\infty$, par rapport ì $\varphi$, de $\varphi=0$ à $\varphi=\frac{\pi}{2}$, et par rapport à $\psi$, de $\psi=0$ ì $\psi=2 \pi$, on aura les trois composantes $X^{\prime \prime} w, Y^{\prime \prime} w$, $Z^{\prime \prime} w$ de laction demandée. On trouve ainsi:

$$
X^{\prime \prime}=A\left(\frac{\partial u}{\partial z}+\frac{\partial w}{\partial x}\right), \quad Y^{\prime \prime}=A\left(\frac{\partial v}{\partial z}+\frac{\partial w}{\partial y}\right), \quad Z^{\prime \prime}=A\left(\frac{\partial u}{\partial x}+\frac{\partial v}{\partial y}+3 \cdot \frac{\partial w}{\partial z}\right),
$$

$A$ représentant ici, comme dans la note première l'expression

$$
\left[\frac{2}{15} \pi \int_{0}^{\infty} \zeta^{t} F(\zeta) \partial \zeta\right] \text {. }
$$

Seconde section. Théorèmes sur les pressions.

21.

Parmi les 9 composantes $X, Y, Z, X^{\prime}, Y^{\prime}, Z^{\prime}, X^{\prime \prime}, Y^{\prime \prime}, Z^{\prime \prime}$ données dans les paragraphes 12. et 13., il est bon de distinguer les composantes $X, Y^{\prime}, Z^{\prime \prime}$, respectivement normales aux élémens plans correspondans, forces que nous désignerons par $N_{1}, N_{2}, N_{3}$, et les six autres composantes égales deux à deux: $Z^{\prime}=Y^{\prime \prime}, X^{\prime \prime}=Z, Y=Z^{\prime}$, que nous nommerons forces tangentielles, et que nous désignerons pareillement par $T_{1}$, $T_{2}, T_{3}$. Les égalités qui précèdent, sont autant de théorèmes remarquables, mais qui ne sont que des cas particuliers d'un théorème plus général qui sera bientôt démontré.

\section{2.}

Si on change d'axes coordonnés, on aura d̀ considérer six nouvelles forces $N_{1}^{\prime}, N_{2}^{\prime}, N_{3}^{\prime}, T_{1}^{\prime}, T_{2}^{\prime}, T_{2}^{\prime}$ On pourra les exprimer en fonc- 
156 19. Lamé et Clapeyron, mérn. sur l'́quilitre int. des corps sol. homog.

tion des premières forces $N_{1}, N_{2}, N_{3}, T_{1}, T_{2}, T_{3}$, et des angles de direction des nouveaux axes relativement aux anciens.

Pour trouver les relations qui existent entre ces systèmes différens, soient $x^{\prime}, y^{\prime}, z^{\prime}$ les nouvelles coordonnées; $m_{1}, m_{2}, m_{3} ; n_{1}, n_{2}, n_{3}$; $p_{1}, p_{2}, p_{3}$ les cosinus des angles que chacune fait avec les axes des $x$, des $y$ et des $z$; enfin soient $u^{\prime}, v^{\prime}, w^{\prime}$ les projections sur les nouveaux axes de l'espace qui sépare une molécule $M$ de la position qu'elle occuperoit, si l'homogénéité du corps n'avait pas été troublée. On aura:

$$
\text { 1. }\left\{\begin{array}{c}
x=m_{1} x^{\prime}+n_{1} y^{\prime}+p_{1} z^{\prime}, \quad y=m_{2} x^{\prime}+n_{2} y^{\prime}+p_{2} z^{\prime}, \\
z=m_{3} x^{\prime}+n_{3} y^{\prime}+p_{3} z^{\prime} \\
u^{\prime}=m_{1} u+m_{2} v_{1}+m_{3} w, v^{\prime}=n_{2} u+n_{2} v+n_{3} w \\
w^{\prime}=p_{1} u+p_{2} v+p_{3} w ;
\end{array}\right.
$$

les cosinus $m_{1}, n_{1}, p_{1} ; m_{8}, n_{2}, p_{2} ; m_{3}, n_{3}, p_{3}$ étant liés entreux par les six équations:

2. $\left\{\begin{array}{l}m_{1}^{2}+n_{1}^{2}+p_{1}^{2}=1, m_{2}^{2}+n_{2}^{2}+p_{2}^{2}=1, m_{3}^{2}+n_{3}^{2}+p_{3}^{2}=1, \\ m_{2} m_{3}+n_{2} n_{3}+p_{2} p_{3}=0 ; m_{1} m_{2}+n_{1} n_{2}+p_{1} p_{2}=0 ; m_{3} m_{1}+n_{3} n_{1}+p_{3} p_{1}=0 .\end{array}\right.$ On déduit des équations (1.) par des différentiations convenables, et en ayant égard aux équations (2.):

$$
\begin{aligned}
& N_{1}^{\prime}=A\left(3 \cdot \frac{\partial u^{\prime}}{\partial x^{\prime}}+\frac{\partial v^{\prime}}{\partial y^{\prime}}+\frac{\partial w^{\prime}}{\partial z^{\prime}}\right)=m_{1}^{2} N_{1}+m_{2}^{2} N_{2}+m_{3}^{2} N_{3}+2 m_{2} m_{3} T_{2}+2 m_{1} m_{3} T_{8} \\
& \begin{array}{r}
N_{2}^{x}=A\left(\frac{\partial u^{\prime}}{\partial x^{\prime}}+3 \cdot \frac{\partial v^{\prime}}{\partial y^{\prime}}+\frac{\partial w^{\prime}}{\partial z^{\prime}}\right)=n_{1}^{2} N_{1}+n_{2}^{2} N_{8}+n_{3}^{2} N_{3}+2 m_{1} m_{2} n_{3} T_{1}+2 n_{1} n_{3} T_{8}
\end{array} \\
& +2 n_{2} n_{2} T_{3} \\
& N_{3}^{x}=A\left(\frac{\partial u^{\prime}}{\partial x^{\prime}}+\frac{\partial v^{\prime}}{\partial y^{\prime}}+3 \cdot \frac{\partial w^{\prime}}{\partial z^{\prime}}\right)=p_{1}^{2} N_{2}+p_{2}^{2} N_{2}+p_{3}^{2} N_{3}+2 p_{3} p_{3} T_{2}+2 p_{1} p_{3} T_{8}^{\prime} \\
& +2 p_{2} p_{2} T_{3} \\
& T_{2}^{x}=A\left(\frac{\partial w^{\prime}}{\partial y^{\prime}}+\frac{\partial v^{\prime}}{\partial z^{\prime}}\right)=p_{2} n_{1} N_{2}+p_{2} n_{2} N_{2}+p_{3} n_{3} N_{3}+\left(p_{2} n_{3}+n_{2} p_{3}\right) T_{1} \\
& +\left(p_{1} n_{3}+n_{1} p_{3}\right) T_{2}+\left(p_{1} n_{2}+n_{2} p_{2}\right) T_{3} \\
& T_{2}^{\mathrm{x}}=A\left(\frac{\partial u^{\prime}}{\partial z^{\prime}}+\frac{\partial w^{\prime}}{\partial x^{\prime}}\right)=m_{1} p_{2} N_{2}+m_{2} p_{2} N_{2}+m_{3} p_{3} N_{3}+\left(m_{2} p_{3}+p_{2} m_{3}\right) T_{1} \\
& +\left(m_{1} p_{3}+p_{1} m_{3}\right) T_{8}+\left(m_{1} p_{8}+p_{2} m_{8}\right) T_{3} \\
& T_{3}^{\mathrm{x}}=A\left(\frac{\partial v^{\prime}}{\partial y^{\prime}}+\frac{\partial u^{\prime}}{\partial x^{\prime}}\right)=n_{1} m_{1} N_{1}+n_{2} m_{2} N_{2}+n_{3} m_{3} N_{3}+\left(n_{2} m_{3}+m_{2} n_{3}\right) T_{2} \\
& \text { Et enfin: } \\
& +\left(n_{8} m_{3}+m_{1} n_{3}\right) T_{8}+\left(n_{1} m_{8}+m_{8} n_{8}\right) T_{3} \text {. }
\end{aligned}
$$

$$
\text { 4. } \frac{\partial u^{\prime}}{\partial x^{\prime}}+\frac{\partial v^{\prime}}{\partial y^{\prime}}+\frac{\partial w^{\prime}}{\partial z^{\prime}}=\frac{\partial u}{\partial x}+\frac{\partial v}{\partial y}+\frac{\partial w}{\partial z}=\theta \text {. }
$$

Cette équation (4.) démontre un théorème que l'on peut enoncer ainsi: En un point quelconque d'un corps solidedont l'homogé- 
19. Lamé te Clapeyron, mém. sur l'équilibre int. des corps sol. homog. 167 néité est troublée, la dilatation cubique est égale à la somme des dilatations linéaires, prises en ce point, dans trois directions quelconques perpendiculaires entr'elles.

Car $\frac{\partial u}{\partial x} \partial x$ peut être regardé comme laugmentation de la ligne $\partial x$ parallèle ì l'axe des $x$, passant par le point $M$, et $\frac{\partial u}{\partial x}$ comme le rapport de cette augmentation ì la ligne elle même, ou comme sa dilatation.

Les équations (3.) donnent immédiatement:

$$
N_{\mathrm{x}}^{\mathrm{x}}+N_{2}^{\mathrm{x}}+N_{3}^{\mathrm{x}}=N_{1}+N_{\mathrm{s}}+N_{3}=5 \mathrm{~A} \theta
$$

d'où résulte cet autre théorème que: la somme des composantes normales des tractions ou pressions exercées sur trois élémens plans, menés perpendiculairement entr'eux, en un méme point du corps solide, est constante, quelque soit lee système decestrois plans, et est de plus égale i la dilatation multipliée par un coëfficient constaht dans toute l'étendue du corps.

23.

Soient $P_{1}, P_{2}, P_{3}$ les tractions ou pressions obliques dont les composantes sont $N_{2}, T_{3}, T_{2} ; T_{3}, N_{2}, T_{1} ; T_{2}, T_{1}, N_{3}$; et soient $\alpha_{1}, \alpha_{2}$, $\alpha_{3} ; \beta_{1}, \beta_{2}, \beta_{3} ; \gamma_{1}, \gamma_{2}, \gamma_{3}$ les angles que ces forces font avec les axes; on aura:

$$
\text { 5. }\left\{\begin{array}{lll}
N_{1}=P_{1} \cos \alpha_{1}, & T_{3}=P_{2} \cos \alpha_{2}, & T_{2}=P_{3} \cos \alpha_{3}, \\
T_{3}=P_{1} \cos \beta_{1}, & N_{2}=P_{2} \cos \beta_{2}, & T_{1}=P_{3} \cos \beta_{3}, \\
T_{2}=P_{1} \cos \gamma_{1}, & T_{1}=P_{2} \cos \gamma_{2}, & N_{3}=P_{3} \cos \gamma_{3} .
\end{array}\right.
$$

Dans ces équations les quantités $N_{\mathrm{r}}, N_{2}^{\prime}, N_{3} ; T_{1}, T_{2}, T_{3}$ données en nombres par les équations des paragraphes 12. et 13., sont ou positives on négatives; leurs grandeurs et leur signes seront connues en chaque point du corps solide, lorsque les fonctions $u, v, w$, correspondantes aux circonstances données, seront déterminées; alors les équations (5.) feront connaître en grandeur et en direction les forces $P_{1}, P_{2}, P_{3}$; suivant qu'elles feront avec les normales qui leur correspondent, ou les axes des $x$, des $y$ et des $z$, des angles surpassant, également l'angle droit, ou étant moindre que lui, ces forces $P_{1}, P_{2}, P_{3}$ seront des pressions des forces tangentielles, ou des tractions.

On conclut des équations (5.):

6. $\quad P_{1}^{2}=N_{1}^{2}+T_{3}^{2}+T_{2}^{2}, \quad P_{2}^{2}=T_{3}^{2}+N_{2}^{2}+T_{2}^{2}, \quad P_{1}^{2}=T_{2}^{2}+T^{2}+N_{3}^{2}$; 
6. $N_{2} T_{3}+N_{2} T_{3}+T_{1} T_{2}=P_{1} P_{2} \cos \left[P_{1}, P_{2}\right] ; N_{1} T_{2}+T_{1} T_{3}+N_{3} T_{2}=P_{2} P_{3} \cos \left[P_{1}, P_{3}\right] ;$

$$
T_{2} T_{3}+N_{8} T_{3}+N_{3} T_{1}=P_{8} P_{3} \cos \left[P_{2}, P_{3}\right]
$$

\section{4.}

Soient pareillement $P_{x}^{x}, P_{2}^{x}, P_{3}^{x}$ les tractions on pressions oblipues aux élémens plans, passant par le point $M$, et parallèles aux nouveaux axes, on aura pour déterminer $P_{x}^{x}$ et sa direction relativement aux anciens axes, les équations:

$$
\begin{aligned}
& P_{x}^{x} \cos \left[P_{x}^{x}, x\right]=m_{1} N_{x}^{x}+n_{x} T_{3}^{x}+p_{x} T_{2}^{x}, \\
& P_{x}^{x} \cos \left[P_{x}^{x}, y\right]=m_{2} N_{x}^{x}+n_{2} T_{3}^{x}+p_{2} T_{2}^{x}, \\
& P_{x}^{x} \cos \left[P_{x}^{x}, z\right]=m_{3} N_{x}^{x}+n_{3} T_{3}^{x}+p_{3} T_{2}^{x} ;
\end{aligned}
$$

or les équations (3.) donnent:

$$
\begin{aligned}
& m_{x} N_{x}^{x}+n_{x} T_{3}^{x}+p_{x} T_{2}^{x}=m_{x} N_{x}+m_{2} T_{3}+m_{3} T_{2}=P_{x} \cos \left[P_{x}, x^{\prime}\right], \\
& m_{2} N_{x}^{x}+n_{2} T_{3}^{x}+p_{2} T_{2}^{x}=m_{x} T_{3}+m_{2} N_{2}+m_{3} T_{x}=P_{2} \cos \left[P_{2}, x^{\prime}\right], \\
& m_{3} N_{x}^{x}+n_{3} T_{3}^{x}+p_{3} T_{2}^{x}=m_{x} T_{2}+m_{2} T_{x}+m_{3} N_{3}=P_{3} \cos \left[P_{3}, x^{\prime}\right] .
\end{aligned}
$$

On a donc simplement

7. $P_{x}^{\mathrm{x}} \cos \left(P_{1}^{\mathrm{x}}, x\right)=P_{\mathrm{x}} \cos \left(P_{1}, x^{\prime}\right) ; P_{\mathrm{x}}^{\mathrm{x}} \cos \left(P_{\mathrm{x}}^{\mathrm{x}}, y\right)=P_{2} \cos \left(P_{2}, x^{\prime}\right)$;

et par suite:

$$
P_{x}^{x} \cos \left(P_{x}^{x}, z\right)=P_{3} \cos \left(P_{3}, x^{\prime}\right) \text {; }
$$

$$
P_{x}^{\mathrm{x}^{2}}=P_{\mathrm{x}}^{2} \cos ^{2}\left[P_{x}, x^{\prime}\right]+P_{2}^{2} \cos ^{2}\left[P_{2}, x^{\prime}\right]+P_{3}^{2} \cos ^{2}\left[P_{3}, x^{\prime}\right]
$$

on trouveroit de la même manière:

$$
\begin{aligned}
& P_{2}^{x^{2}}=P_{1}^{2} \cos ^{2}\left[P_{x}, y^{\prime}\right]+P_{2}^{2} \cos ^{2}\left[P_{2}, y^{\prime}\right]+P_{3}^{2} \cos ^{2}\left[P_{3}, y^{\prime}\right] ; \\
& P_{3}^{x^{2}}=P_{x}^{2} \cos ^{2}\left[P_{x}, z^{\prime}\right]+P_{2}^{2} \cos ^{2}\left[P_{2}, z^{\prime}\right]+P_{3}^{2} \cos ^{2}\left[P_{3}, z^{\prime}\right] ;
\end{aligned}
$$

d'où, en ajoutant les trois équations précédentes:

$$
P_{x}^{x^{2}}+P_{2}^{x^{2}}+P_{3}^{x^{2}}=P_{x}^{2}+P_{2}^{2}+P_{3}^{2} \text {. }
$$

\section{5.}

Les équations (\%.) démontrent ce théorème, que: Si en un même point d'un corps solide homogène, soumis à des forces quelconques, on conçoit deux élémens plans $w$, $w^{\prime}$, ayant pour perpendiculaires $n$ et $n^{\prime}$, les tractions ou pressions, en général obliques $\varpi$, $\varpi^{\prime}$, exercées sur ces plans, jouiront de cette propriété que la composante de w sur $n^{\prime}$, et celle de $w^{\prime}$ sur $n$ seront égales.

L'équation (8.) démontre que: la somme des quarrés des pressions ou tractions exercées sur trois élémens plans rectangulaires, est constante pour un même point du corps so- 
19. L a mé et Cl.ap.eyr.on, mém. sur l'équilibre int. des, corps sol. homag. 169

lide proposé, quelle que soit la position du systême deces trois élémens.

Il suit encore des équations (7.) que si, connoissant en grandeur et en direction, les pressions obliques $\varpi^{\prime}, \varpi^{\prime \prime}, \varpi^{\prime \prime \prime}$, exercées en un point d'un corps solide, sur trois élěmens plans $\omega^{\prime \prime}$, $\omega^{\prime \prime}, \omega^{\prime \prime \prime}$, perpendiculaires entr'eux, et ayant respectivement pour normales les droites $v^{\prime}, v^{\prime \prime}, v^{\prime \prime \prime}$, on veut determiner la grandeur et la direction de la pression $\sigma$ exercée sur un quatrième élément plan $\omega$, ayant pour normale $v$, il faut projeter chacume des pressious comues $\varpi^{\prime}, \varpi^{\prime \prime}$, $\varpi^{\prime \prime \prime}$, sur la normale $\nu$; ces trois projections portées, à partir du point $M$, sur les normales correspondantes $v^{\prime}, v^{\prime \prime}, v^{\prime \prime \prime}$, seront les composantes de la pression ๘ sur ces mêmes droites et la diagonale du parallèlepipède rectangle ayant ces composantes pour cotés, donnera en grandeur et en direction la pression demandée.

(La snite dans le cahier prochain.) 